\title{
Geometric constraints alter the emergent dynamics of an active particle
}

\author{
Aniruddh Murali $\odot,{ }^{1, *}$ Pritha Dolai, ${ }^{2, *}$ Ashwini Krishna $\odot,{ }^{1}$ K. Vijay Kumar $\odot,{ }^{2, \dagger}$ and Shashi Thutupalli ${ }^{1,2,+}$ \\ ${ }^{1}$ Simons Centre for the Study of Living Machines, National Centre for Biological Sciences, Tata Institute of Fundamental Research, \\ GKVK Campus, Bellary Road, Bengaluru 560065, India \\ ${ }^{2}$ International Centre for Theoretical Sciences, Tata Institute of Fundamental Research, Survey No. 151, Shivakote, \\ Hesaraghatta Hobli, Bengaluru 560089, India
}

(Received 12 May 2021; accepted 24 January 2022; published 18 February 2022)

\begin{abstract}
A self-propelled particle is a basic ingredient of active matter. Any handle on its noise characteristics is thus of both fundamental and applied interest. In this paper, we show that geometric constraints are a route to affect the emergent noise properties of a single active particle, thus demonstrating that seemingly different active particle classes are equivalent to each other. Specifically, we find that the chiral active Brownian motion of a self-propelled particle seen in two-dimensions switches to a run-and-tumble like motion when confined to a quasi-one-dimensional channel. Our analysis of this switching behavior connects the resulting active stochastic dynamics to that of a two-state molecular motor. The emergent tuning of active noise characteristics by unbiased external driving, as we demonstrate here, is illustrative of generic mechanisms of active noise control in other systems.
\end{abstract}

DOI: 10.1103/PhysRevResearch.4.013136

\section{INTRODUCTION}

A hallmark of self-organization in non-equilibrium systems is the generation of ordered states in a controlled manner driven by external energy input [1]. This organization often appears due to the spontaneous rectification of environmental fluctuations that otherwise destabilise the appearance of such ordered states. In biological cells, for example, individual molecular motors can utilize unbiased energy input and rectify ambient stochasticity to generate directed motion while cells themselves can control their direction of motion by responding to fluctuating external cues. Such emergent order is also seen in many synthetic systems: for example, (i) the generation of persistent motion of a rotational ratchet embedded in a granular gas of macroscopic glass beads that is driven by mechanical agitation [2], or (ii) directed self-propelled motion of Janus colloids with differential chemical reactions occurring on their surfaces [3,4]. Indeed, the harnessing of noise to generate ordered states is a fundamental tenet in many non-equilibrium systems.

Active materials are a generic class of non-equilibrium systems in which driving and dissipation occur at the level of a individual unit [5-7]. The fundamental units of active mattertypically self-propelled particles-display athermal motion driven by stochastic forces arising from the broken detailedbalance in the system. Altering the characteristics of this

\footnotetext{
${ }^{*}$ These authors contributed equally to this work.

†vijaykumar@icts.res.in

"shashi@ncbs.res.in
}

Published by the American Physical Society under the terms of the Creative Commons Attribution 4.0 International license. Further distribution of this work must maintain attribution to the author(s) and the published article's title, journal citation, and DOI. "active noise", therefore, can change the qualitative nature of the emergent self-organization in these systems. Noise-control in active matter, i.e., tuning the spatiotemporal distribution of the driving forces, has been of growing interest recently [8-11]. Additionally, controlling noise characteristics in an active system can lead to efficient ways of sampling the possible microscopic states that underlie an ordered macroscopic state [12]. Given that the active matter framework has been of relevance in living matter [13-16] and a new class of synthetic materials [17-21], controlling active noise opens up novel possibilities for self-organized phenomena in these systems.

In synthetic active matter systems, self-propelled droplets [22,23], colloidal systems [3,24], and granular media [25,26], provide experimental realisations of "scalar active matter"self-propelled particles wherein the interactions between individual particles do not depend on their respective orientations [27]. Theoretical models of scalar active matter are typically of three broad types - the essential distinguishing feature of these is the characteristics of the active noise driving the self-propelled motion. (i) Active Brownian particles (ABP), exemplified by the motion of self-phoretic colloids $[3,24,28]$ and driven granular particles [25,26], move with a constant speed with their motility direction undergoing rotational diffusion. (ii) Run-and-tumble-particles (RTP), inspired by the swimming motion of bacteria [29], also move with a constant speed along a given direction for a certain "runtime", after which they undergo an instantaneous "tumble" that randomises their direction of motion; the run-times are governed by a characteristic Poisson process [30]. (iii) The dynamics of active Ornstein-Uhlenbeck particles (AOUP) are driven by a Gaussian noise with a finite-correlation time [31].

The models of scalar active matter outlined above have been used to understand self-propelled motion in disparate experimental systems. However, it is an open question if these are fundamentally different classes of active particles, or if 


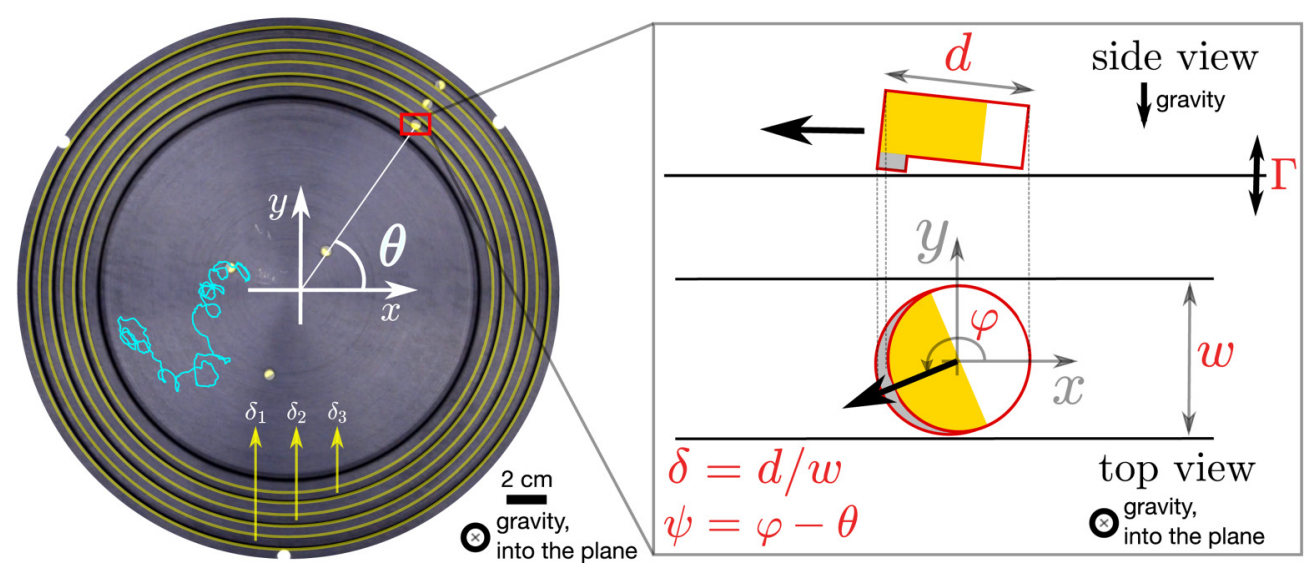

FIG. 1. Experimental setup: A circular 3D printed granular particle of diameter $d$ is driven using an electromagnetic shaker (nondimensional acceleration $\Gamma$ ). The particle displays active self-propelled motion (blue trajectory) due to frictional asymmetry with the substrate. This two-dimensional motion is well captured by an ABP model describing a chiral particle with position $\mathbf{r}$ and orientation $\varphi$. Quasi-onedimensional confinement of the particle is achieved using concentric circular channels of varying widths $w$ (confinement $\delta=d / w$ ). This one-dimensional motion is tracked using the coordinate $\theta$ and the relative internal coordinate $\psi$.

switching between these broad types of active dynamics is possible within a single experimental setup. Note that a direct access to the noise characteristics in an active system can be achieved by biasing the input that drives its dynamics. For instance, explicitly modulating the external driving of the reorientation dynamics of self-propelled particles has recently been implemented to tune the qualitative nature of their persistent random walks [8-11]. Instead, we ask: Can such tuning of the active dynamics emerge via an unbiased energy input? We show that a subtle coupling between the internal degrees of a self-propelled particle and the external constraints acting upon it, can indeed switch the qualitative nature of the active dynamics. In a broader context, such coupling mechanisms could underlie the motility of self-propelled objects, such as cells, due to the geometrical characteristics of their environment [32].

We use an experimental system comprised of a driven granular particle to show that laterally confining a chiral ABP-like particle in a narrow quasi-one-dimensional channel leads to the emergence of RTP-like motion, and that the characteristics of this active noise can be controlled by our empirical parameters. To understand the geometrically induced dynamics, we model the effect of the confining walls as a torque on the orientation of the chiral active particle, and show that this simple treatment captures both the steady-state and dynamical characteristics of the emergent RTP motion. A natural connection then arises between the dynamics of our active particle and that of a molecular motor modelled by Brownian motion in a periodic potential subject to a constant forceindeed, it is the chirality of our granular particle that maps to such a constant force. Our results are a clear demonstration of confinement-induced tuning of the noise characteristics of active particles, and suggest ways to harness their dynamics.

\section{EXPERIMENTAL SETUP}

Our experimental system is comprised of circular 3D printed disks (particles) of diameter $d$, with an asymmetric leg on one side (Fig. 1 and Supplemental Material [SM] [33]); when placed on a flat surface (aluminum disk) and driven using an electromagnetic shaker (see SM [33]), the particles propel in-plane along a fixed body axis. The shaker imparts a periodic (sinusoidal) driving in a plane perpendicular to the aluminium disk-the frequency and amplitude of this periodic driving can be tuned and is captured by the nondimensional acceleration $\Gamma=A \Omega^{2} / g$, where $A$ is the maximum vertical displacement of the disk, $\Omega$ is the shaking frequency and $g=9.81 \mathrm{~m} / \mathrm{s}^{2}$ is the acceleration due to gravity. Owing to its design, a shape anisotropy is evident in the 2-dimensional projected diameter of the particle along the propulsion direction (Fig. 1). While the asymmetry in the particle is front-back by design, manufacturing defects cause slight (uncontrolled) asymmetries in the axial direction due to which the particle motion is chiral, as we describe later. In unconfined two-dimensional space, the particle is an active, motile object executing a self-propelled random walk (Movie 1 in SM [33]) with continuously and noisily varying position $\mathbf{r}=(x, y)$ and orientation $\varphi$ (Fig. 1 and SM [33]). In addition to self-propulsion, the particles exhibit chiral motion characterized by an angular speed $\omega$. Altogether, the motion of the active particle in the unconfined 2-dimensional space is well characterized by an ABP model [26] for a chiral active particle (see MSD analysis in the SM [33]): The particle position evolves according to $\dot{\mathbf{r}}=v_{0} \hat{\mathbf{e}}(\varphi)+\sqrt{2 D_{t}} \boldsymbol{\eta}(t)$ where $\hat{\mathbf{e}}(\varphi)=\cos \varphi \hat{\mathbf{x}}+\sin \varphi \hat{\mathbf{y}}$ is the local direction of motion, the over-dot indicates a time-derivative, $v_{0}$ is an active speed, $D_{t}$ is the translational diffusion coefficient, and the stochastic term $\eta(t)$ is a Gaussian white-noise process with zero mean and unit variance. The angular coordinate $\varphi$ of the ABP performs a random walk governed by $\dot{\varphi}=\omega+\sqrt{2 D_{r}} \zeta(t)$ with $\omega$ the (chiral) angular velocity, $D_{r}$ the rotational diffusion coefficient of the ABP while $\zeta(t)$ is a zero-mean and unit-variance Gaussian white-noise process. In our experimental set-up, we are able to control these various parameters of the ABP model, viz., $\omega, D_{r}, v_{0}$, and $D_{t}$, using the driving $\Gamma$ of the electromagnetic shaker (see SM [33]). 

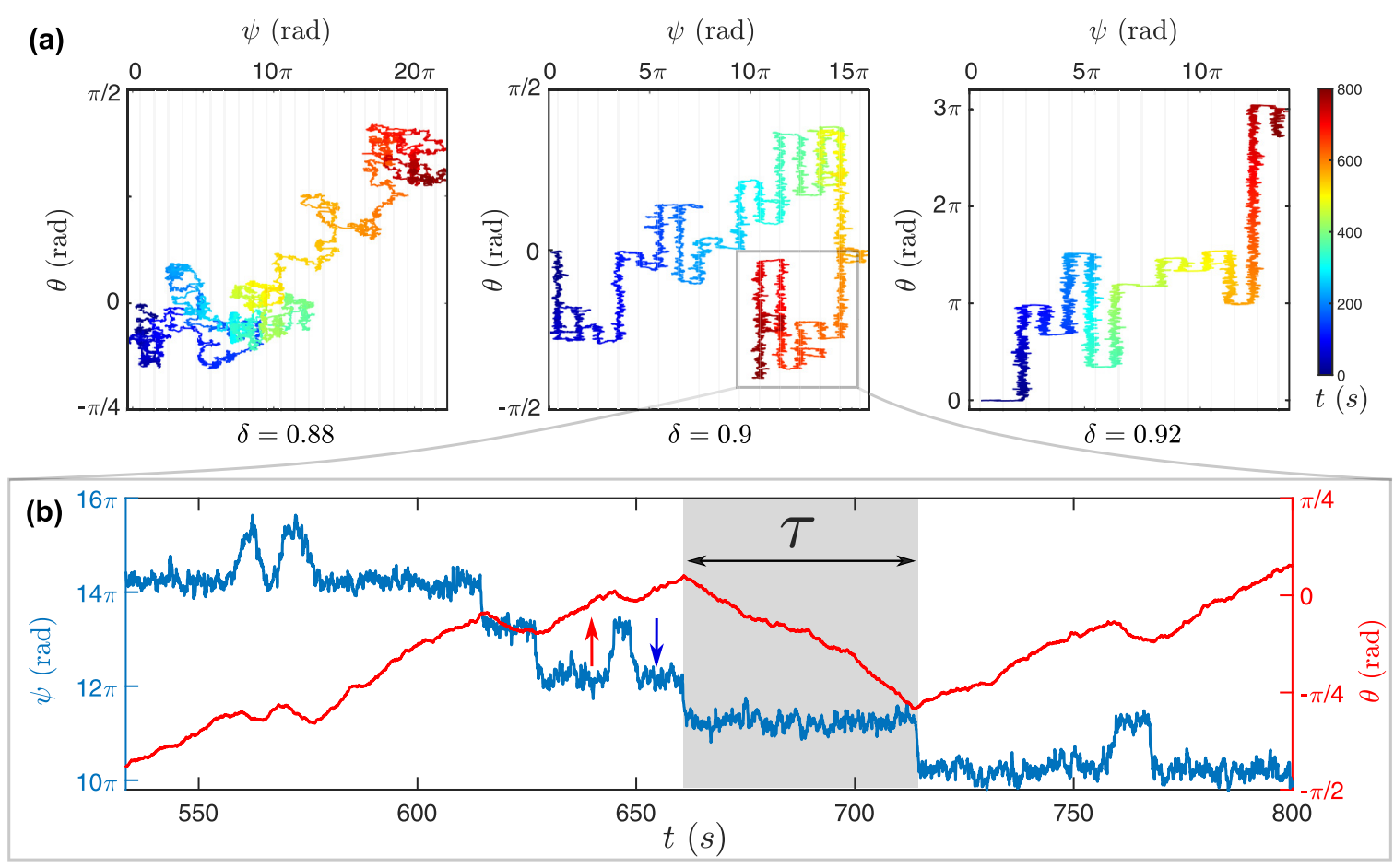

FIG. 2. Emergent run-and-tumble dynamics in quasi-one-dimensional confinement. (a) Parametric plot of the particle trajectories in the translational co-ordinate $\theta$ and the relative orientation co-ordinate $\psi$ for varying confinements $\delta$, with vertical grid lines marking multiples of $\pi / 2$. Discrete jumps in $\psi$ are seen at sufficiently high $\delta$. A constant drift of $\psi$, due to the particle chirality, to the right ("downhill” direction) is evident. (b) The particle trajectory in $\theta$ is reminiscent of that of a run-and-tumble particle motion with a run duration $\tau$. Red and blue arrows on the trajectory mark particle flips in the "uphill" and "downhill" directions respectively.

\section{EMERGENT STOCHASTIC SWITCHING DYNAMICS IN CONFINEMENT}

We now turn our attention to the behavior of these active particles when they are confined to quasi-one-dimensional channels—grooves of width $w$ that run along the periphery of the aluminum disk as concentric circles (Fig. 1). The geometric configuration of the channels is such that the particles are constrained radially to within $\leqslant 1 \%$ of the channel radius (see SM [33]) and can still explore all possible values of $\varphi$. When the confinement $\delta=d / w \approx 0.88$ is sufficiently small (i.e., a wide channel) the orientation coordinate $\psi$ samples all directions uniformly $[P(\psi)$ for various confinements $\delta$ are not shown] and since this co-ordinate is coupled to the position co-ordinate $\theta$, the particle executes a one-dimensional persistent random walk along the channel (Fig. 2(a), left panel; Movie 2 in SM [33]). The chirality of particle is evident in the steady drift of the orientation $\psi$.

A striking difference is manifest when $\delta$ is increased to $\approx 0.9$ : instead of evolving continuously, the orientation of the particle fluctuates noisily along one of the channel directions i.e., $\psi= \pm \pi / 2$, only to stochastically and abruptly switch direction [Fig. 2(a), middle panel]. The discrete orientation jumps $|\Delta \psi| \sim \pi$ occur either "downhill" in a direction dictated by the chirality of the particle or less frequently "uphill" [blue and red arrows, respectively in Fig. 2(b)]. As the confinement $\delta$ is further increased to $\approx 0.92$, the switches in the particle orientation become much less frequent-in the particular run shown [Fig. 2(a), right panel], they occur only "downhill". When $\psi \sim \pm \pi / 2$, the particle executes a "run" along the channel for a typical "run-time" duration $\tau$ until it stochastically switches to a "run" in the opposite direction (Fig. 2(b); Movie 3 in SM [33]).

Taken together, the characteristics discussed above are reminiscent of a run-and-tumble particle in one-dimension, with position co-ordinate $\theta$. As such, the relevant equation describing the dynamics of $\theta$ is that of an ABP confined to move on a circle of radius $R$ (see SM [33]), viz.,

$$
\dot{\theta}=v \sin \psi+\sqrt{2 \mathcal{D}} \xi(t) \equiv v \sigma(t)+\sqrt{2 \mathcal{D}} \xi(t),
$$

where $v=v_{0} / R, \mathcal{D}=D_{t} / R^{2}, \xi(t)$ is a Gaussian whitenoise process with zero mean and unit variance, and $\sigma(t)=$ $\sin [\psi(t)]$ is the active noise. This model is validated by first computing the empirical two point correlation $C(\mid t-$ $\left.t^{\prime} \mid\right)=\left\langle\sigma(t) \sigma\left(t^{\prime}\right)\right\rangle$ of the active noise, assuming $\sigma(t)$ is a stationary stochastic process. The correlation function decays exponentially with a time-constant $\tau_{\theta} \sim 9 s$ [Fig. 3(a)]. For $\psi \sim \pm \pi / 2$, the active noise $\sigma(t) \sim \pm 1$, and thus $v$ would correspond to the active speed of the RTP-like motion for $\theta$. The bounded values $\sigma(t) \in[-1,1]$ and the exponential decay of $C\left(\left|t-t^{\prime}\right|\right)$ thus justify the effective dynamics of $\theta$ in Eq. (1) as the position coordinate of an RTP-like particle.

We next compare the empirically measured mean-squareddisplacement (MSD) $\left\langle[\Delta \theta(t)]^{2}\right\rangle$ with that predicted from Eq. (1), using the values of $v_{0}$ and $D_{t}$ inferred from the twodimensional experiments for the ABP model:

$$
\left\langle[\Delta \theta(t)]^{2}\right\rangle=v^{2} \int_{0}^{t} d z \int_{0}^{t} d z^{\prime} C\left(\left|z-z^{\prime}\right|\right)+2 \mathcal{D} t .
$$



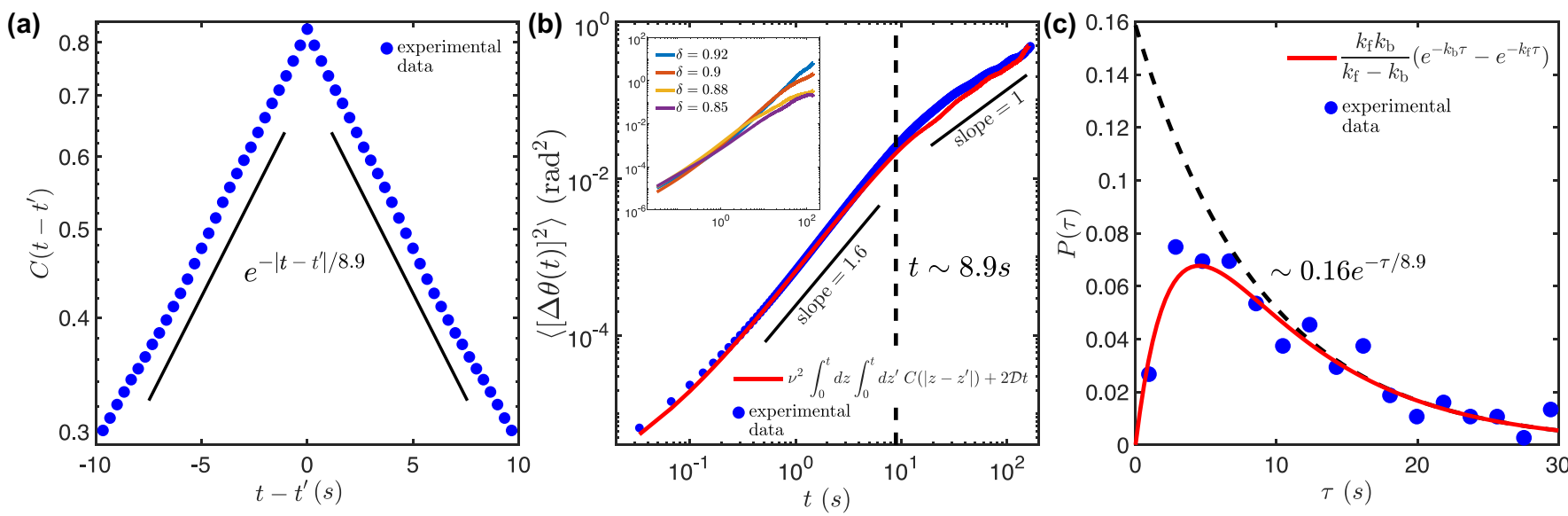

FIG. 3. Characteristics of the run-and-tumble dynamics. (a) Two-point correction function $C\left(\left|t-t^{\prime}\right|\right)=\left\langle\sigma(t) \sigma\left(t^{\prime}\right)\right\rangle$ where $\sigma(t)=$ $\sin [\psi(t)]$ is the active noise. (b) The empirical mean-squared-displacement $\left\langle[\Delta \theta(t)]^{2}\right\rangle$ follows the RTP model in Eq. (1). MSDs for varying confinements are shown in the inset. (c) The run-time distribution $P(\tau)$ of $\theta$, equivalently the dwell-time distribution of $\psi$, exhibits two-time scales fit by the convolution of the statistics of two Poisson processes [Eq. (3)]. In all the plots, blue symbols indicate experimental data and theoretical curves are shown in red.

An excellent agreement is found between the empirical MSD and that given by Eq. (2) [Fig. 3(b)]. Of note in the MSD are the crossovers from an initial diffusive regime (governed by passive translational diffusion), to a super-diffusive regime (governed by the self-propelled motion of the particle), and then, eventually, to a diffusive regime for time-scales $t \gg$ $\tau_{\theta}(\sim 9 \mathrm{~s})$ corresponding to the decorrelation of the persistent motion driven by the active noise $\sigma(t)$. The effective diffusion coefficient in this asymptotic regime has an active contribution $\sim v^{2} \tau_{\theta}$ in addition to the (angular) translational diffusion coefficient $\mathcal{D}[34,35]$. This additional contribution to the effective diffusivity could dramatically increase with increasing confinement as the particle becomes more RTPlike. Indeed, the effective diffusion coefficient does increase with confinement, and in fact for the highest confinements, a crossover to the eventual diffusive regime is not seen on the timescale of our experiments [Fig. 3(b), inset]. These results conclusively suggest that Eq. (1) is indeed a good descriptor for the dynamics of $\theta$, and also that the parameters describing the motion in confinement remain reasonably similar to the particle motion in two-dimensions (more details in the SM [33]).

The empirically measured dwell time distribution of $\psi$ (equivalently, the run time distribution of $\theta$ ) distribution of $\psi$ is well fit by a bi-exponential function

$$
P(\tau)=\frac{k_{\mathrm{f}} k_{\mathrm{b}}}{k_{\mathrm{f}}-k_{\mathrm{b}}}\left(e^{-k_{\mathrm{b}} \tau}-e^{-k_{\mathrm{f}} \tau}\right)
$$

where $k_{\mathrm{f} / \mathrm{b}}$ are the forward ("downhill") and backward ("uphill") rates of the switching between the states $\psi \sim \pm \pi / 2$ [Fig. 3(c)]. It is clear from the above expression that asymptotically, i.e., for $k_{\mathrm{f}} \tau \gg 1$ and $k_{\mathrm{f}} \gg k_{\mathrm{b}}$, the dwell-time distribution $P(\tau) \sim k_{\mathrm{b}} e^{-k_{\mathrm{b}} \tau}$, decaying exponentially with the rate $k_{\mathrm{b}}$. For the same driving strength $\Gamma$, the time-scales that govern the decay of the two-point correlation $C\left(\left|t-t^{\prime}\right|\right)$ and the crossover of the MSD $\left\langle[\Delta \theta(t)]^{2}\right\rangle$ from the super-diffusive to the diffusive regime are comparable to the backward ("uphill") switching rate, i.e., $1 / k_{\mathrm{b}} \sim \tau_{\theta}$.
Several remarks are in order. First, the tumble rate for the RTP motion along the channel, i.e. the transitions between the states $\sigma \sim \pm 1$, is $k_{\mathrm{f}}+k_{\mathrm{b}}$. However, it should be noted that unlike the standard RTP model with an exponentially distributed run time between tumbles, our active particle dynamics demonstrates a non-monotonic distribution $P(\tau)$ of run-times $\tau$. Second, the discrete jumps in the trajectory of $\psi$ [Fig. 2(b)] are reminiscent of the positions of a molecular motor stepping along a polymeric track [36,37]. Third, the fit of Eq. (3) [Fig. 3(c)] suggests the presence of (at least) two characteristic switching rates in the RTP-like dynamics of $\theta$ driven by the active noise $\sigma(t)$. Incidentally, Eq. (3) is used to model the dwell time distributions of a two-state molecular motor [37-39]. All the above points hint at a possible similarity between the dynamics of our active particle in confinement and those of a processive molecular motor. We next show that this is indeed the case.

\section{ANALOGY TO THE STEPPING DYNAMICS OF A MOLECULAR MOTOR}

We conjecture that the effects of the confining channel on the particle can be captured by a periodic torque $T_{\text {wall }}=$ $\lambda \sin 2 \psi$, on the particle orientation, where $\lambda$ is the strength of the wall-particle interaction. In doing this, we simplify the interactions, which are quite complicated indeed-they not only depend on the particle and channel surface roughness and the resultant friction, but possibly also on the amplitude of driving $\Gamma$. However, the step-like trajectories, and the preponderance of the values of $\psi$ close to $\pm \pi / 2$, motivate us to test this functional form of $T_{\text {wall }}$ to describe the particle motion. Following this intuition, the dynamics of $\psi$ is modelled by the following equation (details in SM [33]):

$$
\dot{\psi}=-V^{\prime}(\psi)+\sqrt{2 D} \bar{\xi}(t)
$$

where $D=D_{t} / R^{2}+D_{r}$ with $R$ the (mean) radius of the confining channel, $\bar{\xi}$ is a zero-mean unit-variance Gaussian white noise process, and the prime denotes differentiation with 

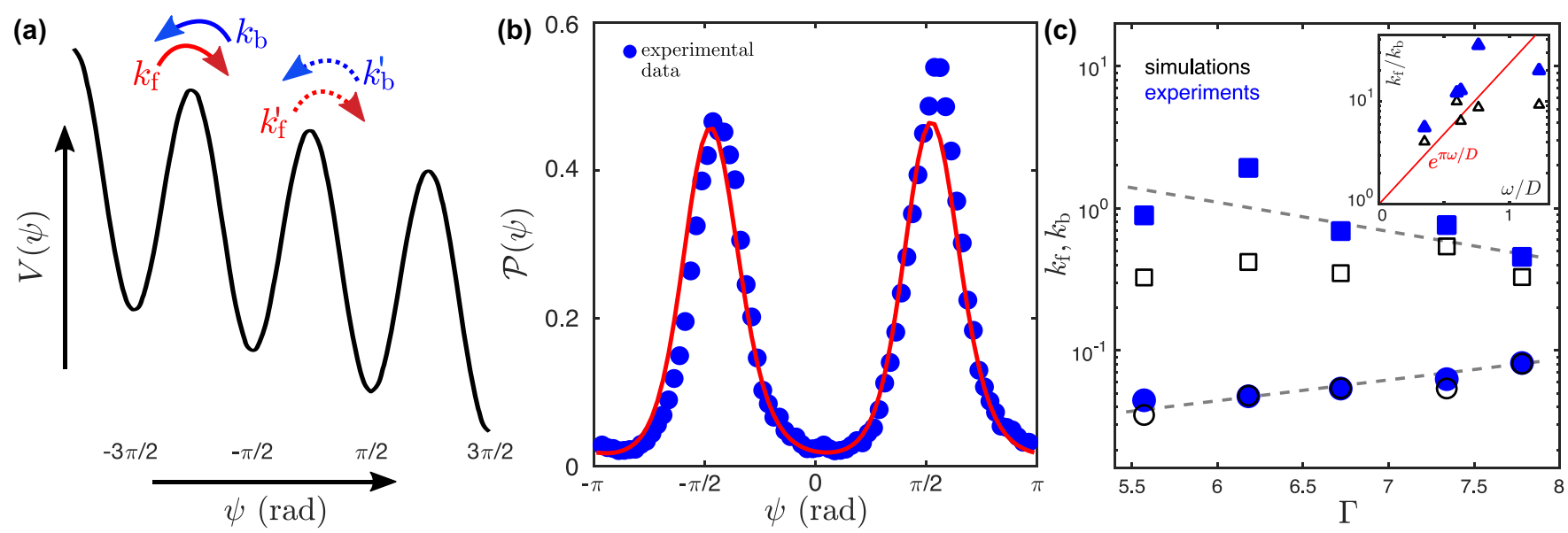

FIG. 4. Dynamics and control of the stochastic switching. (a) Effective potential for the internal co-ordinate $\psi$ and the transition rates between the neighboring minima of the potential. In our analysis, $k_{\mathrm{f} / \mathrm{b}}^{\prime} \approx k_{\mathrm{f} / \mathrm{b}}$. (b) The empirical steady-state distribution $\mathcal{P}(\psi)($ data points) compared with the analytical solution obtained from the Fokker-Planck equation for the stochastic dynamics of $\psi$ (red line). (c) Control of the forward and backward switching rates, $k_{\mathrm{f}}$ and $k_{\mathrm{b}}$ by modulating the driving $\Gamma$. Dashed lines are a guide to the eye. Simulations are performed with parameters extracted using a heuristic fitting procedure (SI Text). The inset shows the ratio $k_{\mathrm{f}} / k_{\mathrm{b}}$ and its correspondence with the prediction from Kramers' rate theory analysis (see SM [33]). In all the plots, blue symbols indicate experimental data, open black symbols indicate simulations and theoretical curves are shown in red.

respect the argument of its function. The effective potential

$$
V(\psi)=-\omega \psi+\frac{\lambda}{2} \cos 2 \psi-v \cos \psi
$$

thus governs the dynamics of $\psi$. Notice that $U(\psi)=V(\psi)+$ $\omega \psi$ is a periodic function of $\psi$. Thus, the Langevin equation (4) describes the stochastic dynamics of a Brownian particle with a position coordinate $\psi$ moving in a periodic potential $U$, subject to a constant "force" $\omega$ [Fig. 4(a)]. It is straightforward to solve the Fokker-Planck equation for $\mathcal{P}(\psi)$ in the steady-state, corresponding to this motion (see SM [33]). This theoretical $\mathcal{P}(\psi)$ agrees well with the empirical distribution, relying on a single fitting parameter $\lambda$ (Fig. 4(b), SM [33]). However, $\lambda$ depends on the driving $\Gamma$ (see SM [33]), which is altogether not surprising given that it encapsulates the effective strength of the complicated interactions between the active particle and the confining channel.

Having concluded that the effective potential $V(\psi)$ is sufficient to capture the steady-state distribution of the internal coordinate $\psi$, we next show that it can also capture the dynamical properties of $\psi$. The two point correlation function $C(\mid t-$ $\left.t^{\prime} \mid\right)$ computed from simulations of the Langevin equation (4) compares well with empirical measurements (see SM [33]). Further, the switching rates, $k_{\mathrm{f}}$ and $k_{\mathrm{b}}$, as inferred from the fits of Eq. (3) to the run-time distributions obtained from both the experimental and simulation data compare well. These rates and other characteristics of the RTP-like motion can be controlled by the driving amplitude $\Gamma$ (Fig. 4(c) and SM [33]). The transition rates governing the jumps of $\psi$ across successive minima of the potential can be explicitly computed in the Kramers' approximation [40]. When $v \neq 0$ there are in principle four transition rates, $k_{\mathrm{f} / \mathrm{b}}$ and $k_{\mathrm{f} / \mathrm{b}}^{\prime}$, across the potential minima ([Fig. 4(a)]; however, in the approximation $v \approx 0$ (valid for our experiments, see SM [33]), only two transition rates $k_{\mathrm{f} / \mathrm{b}}$ remain. The variation of the ratio $k_{\mathrm{f}} / k_{\mathrm{b}}$ from the empirical (experimental and simulation) data compares well with the analytical results obtained in the Kramers' approximation [Fig. 4(c), inset]. Further, in analogy with two-state dynamics of molecular motors, the chirality of our active particle makes the forward and reverse transition rates different, concomitant with the bi-exponential distribution of $P(\tau)$ (see SM [33]). Taken together, the effective potential used to describe the confining effects of the channel is a good predictor for the dynamical properties of $\psi$.

\section{DISCUSSION AND CONCLUSIONS}

By confining a self-propelled granular particle to a quasione-dimensional channel, we have demonstrated emergent active noise properties, qualitatively distinct from those of the two-dimensional unconfined motion. While the twodimensional dynamics is well described by an ABP model, RTP-like behavior emerges due to lateral confinement. This dynamics of the translational coordinate $\theta$ is driven by the internal coordinate $\psi$ of the particle, which in turn has similarities to the stepping dynamics of a molecular motor; most notably, this manifests in the nonmonotonic nature of the dwell-time distribution of $\psi$, which is identical to that found in two-state processive motors. To complete the picture and capture the dynamics of $\psi$, we reduced the effects of confinement to a simple periodic torque, thus mapping it to the dynamics of a Brownian particle moving in a periodic potential subject to a constant external force, which is sufficient to capture the statistical features of the emergent dynamics. Note that our experimental system is athermal, far from equilibrium, and the various interactions between the granular surfaces are complicated, thus possibly requiring a priori, a dynamical description more complex than that given by Eqs. (4) and (5). Therefore, the agreement we find between empirical measurements and the theoretical analysis, in particular the Kramers' transition rates is remarkable. 
We conclude with a few comments. First, our experiment is an explicit demonstration of the inseparable coupling between the internal degrees of freedom and the translational co-ordinates of an active particle [Fig. 2(a)], and also underscores that the equivalence between ABP and RTP dynamics observed at the many-particle level [30] is true even at the single particle level. Second, the chirality of the ABP particle is not a requirement for the emergence of RTP-like motion in the channel. The effects of chirality manifest as distinct forward and backward rates $\left(k_{\mathrm{f}} \neq k_{\mathrm{b}}\right)$ for the directional (clockwise/anticlockwise) switching of $\psi$, while an achiral particle would have $k_{\mathrm{f}}=k_{\mathrm{b}}$. As such, the resulting run (dwell) time distribution of $\theta(\psi)$ is the convolution of the waiting time distributions of two composite Poisson processes with rates $k_{\mathrm{f}}$ and $k_{\mathrm{b}}$ (Fig. 3). Further, the ratio $k_{\mathrm{f}} / k_{\mathrm{b}}$ is controlled by the particle chirality $\omega$ as calculated from Kramers' transition rate theory (Fig. 4). Third, the RTP-like discrete dynamics that we describe here is not directly imposed, for example, using explicit external mechanisms $[8,10,11]$, but is rather an emergent behavior resulting from the geometric constraints on the internal coordinate of the particle. Fourth, the nature of such geometric constraints does indeed matter for the resultant dynamics. For instance, confining the three-dimensional motion of an ABP between two-parallel plates does not, in general, lead to RTP-like motion in two-dimensions, even though the active noise characteristics would change. However, confining the three-dimensional motion of an ABP to a cylinder does indeed lead to RTP-like dynamics - our experiment is a twodimensional realization of this situation. Finally, the interplay between geometry and motility that we have uncovered here may be relevant in more broader situations such as cell motility in complex, three-dimensional environments [32,41,42] and in other synthetic active systems such as catalytic or other Janus colloids in confined geometries [3,24]. Altogether, our paper opens up an avenue to exploit the effects of geometric constraints and non-equilibrium driving to control the collective noise characteristics of interacting active particles.

\section{METHODS}

\section{A. Experiments}

A Brüel \& Kjær electromagnetic shaker (LDS V406) is used to excite a patterned aluminum disk on which the particles move. The shaker is suspended on a trunnion mount designed to isolate mechanical vibration. The aluminium disk measures $25 \mathrm{~cm}$ in diameter and $19 \mathrm{~mm}$ in thickness. Circular channels of varying widths, viz., $5.3 \mathrm{~mm}, 5.1 \mathrm{~mm}$, and $4.9 \mathrm{~mm}$ are precision milled into the disk. The channels are $5 \mathrm{~mm}$ in depth with a mean circular radius of $115.7 \mathrm{~mm}, 105.5 \mathrm{~mm}$ and
$95.5 \mathrm{~mm}$ (from outer perimeter of the disk inwards), leaving a circular 2D arena of radius $88 \mathrm{~mm}$ and depth of $5 \mathrm{~mm}$ in the center of the disk. A channel of width $5.0 \mathrm{~mm}$ and $116.5 \mathrm{~mm}$ mean radius are etched on a separate head from which the corresponding experiments are reported. The aluminium disk is polished followed by soft anodizing to provide a uniform black background and is electrically grounded. Particles are designed in-house and fabricated using a 3D printer (Form2 SLA 3-D printer, resolution $\sim 25 \mu \mathrm{m})$. Particles are $4.5 \mathrm{~mm}$ in diameter $(d)$ and $2.5 \mathrm{~mm}$ in height, which includes a leg of height $0.5 \mathrm{~mm}$. The front half of the particle is marked using yellow paint to identify particle orientation.

\section{B. Image processing and analysis}

Particle dynamics are recorded at $60 \mathrm{fps}$ for a duration of 10-20 minutes using a Nikon DSLR camera aligned above the center of aluminum disk. Images are processed using custom written MATLAB code. From the image analysis, we extract time-series of both the position coordinate $\mathbf{r}$ and the angular orientation $\varphi$ of the active particle (see SM [33]). We calculate $\left\langle[\Delta \mathbf{r}]^{2}\right\rangle$ the positional mean-squared-displacement and $\left\langle[\Delta \varphi]^{2}\right\rangle$ the orientation mean-squared-displacement, where $\langle\cdots\rangle$ represents an average over several realizations, and particles. We then used a Bayesian inference procedure to extract the parameters $\omega, D_{r}, v_{0}, D_{t}$ using the empirically measured MSD (see SM [33]).

\section{Simulations}

We performed Langevin simulations of the Eqs. (1) and (4) using an Euler-Maruyama method with a constant time-step $\Delta t=10^{-3} \mathrm{~s}$ and averaged over $N=10^{4}$ realizations of the noise to construct statistical measures.

\section{ACKNOWLEDGMENTS}

We thank Madan Rao, Stephan Herminghaus, Abhishek Dhar, and Sriram Ramaswamy for comments on the manuscript. We acknowledge support from the Department of Atomic Energy, Government of India, under Projects No. RTI4001 and No. RTI4006, the Simons Foundation (Grant No. 287975 to S.T.) and the Max Planck Society through a Max-Planck-Partner-Group at NCBS-TIFR (S.T.), the Department of Biotechnology, India, through a Ramalingaswami re-entry fellowship (K.V.K.) and by the Max Planck Society through a Max-Planck-Partner-Group at ICTS-TIFR (K.V.K.). We thank Pawan Nandakishore for help with the initial set-up and experiments. P.D. thanks Arghya Das for many useful discussions. S.T. and K.V.K. acknowledge discussions during the KITP 2020 online program on Symmetry, Thermodynamics and Topology in Active Matter.
[1] M. Cross and H. Greenside, Pattern Formation and Dynamics in Nonequilibrium Systems (Cambridge University Press, Cambridge, 2009).

[2] P. Eshuis, K. van der Weele, D. Lohse, and D. van der Meer, Experimental Realization of a Rotational Ratchet in a Granular Gas, Phys. Rev. Lett. 104, 248001 (2010).
[3] J. R. Howse, R. A. L. Jones, A. J. Ryan, T. Gough, R. Vafabakhsh, and R. Golestanian, Self-Motile Colloidal Particles: From Directed Propulsion to Random Walk, Phys. Rev. Lett. 99, 048102 (2007).

[4] W. F. Paxton, A. Sen, and T. E. Mallouk, Motility of Catalytic Nanoparticles through self-generated forces, Chem. - Eur. J. 11, 6462 (2005). 
[5] S. Ramaswamy, The mechanics and statistics of active matter, Annu. Rev. Condens. Matter Phys. 1, 323 (2010).

[6] M. C. Marchetti, J. F. Joanny, S. Ramaswamy, T. B. Liverpool, J. Prost, M. Rao, and R. A. Simha, Hydrodynamics of soft active matter, Rev. Mod. Phys. 85, 1143 (2013).

[7] C. Bechinger, R. Di Leonardo, H. Löwen, C. Reichhardt, G. Volpe, and G. Volpe, Active particles in complex and crowded environments, Rev. Mod. Phys. 88, 045006 (2016).

[8] G. Vizsnyiczai, G. Frangipane, C. Maggi, F. Saglimbeni, S. Bianchi, and R. Di Leonardo, Light controlled 3D micromotors powered by bacteria, Nat. Commun. 8, 15974 (2017).

[9] J. Arlt, V. A. Martinez, A. Dawson, T. Pilizota, and W. C. K. Poon, Painting with light-powered bacteria, Nat. Commun. 9, 768 (2018)

[10] H. Karani, G. E. Pradillo, and P. M. Vlahovska, Tuning the Random Walk of Active Colloids: From Individual Run-AndTumble to Dynamic Clustering, Phys. Rev. Lett. 123, 208002 (2019).

[11] M. A. Fernandez-Rodriguez, F. Grillo, L. Alvarez, M. Rathlef, I. Buttinoni, G. Volpe, and L. Isa, Feedback-controlled active Brownian colloids with space-dependent rotational dynamics, Nat. Commun. 11, 4223 (2020).

[12] Z. Zeravcic, V. N. Manoharan, and M. P. Brenner, Colloquium: Toward living matter with colloidal particles, Rev. Mod. Phys. 89, 031001 (2017).

[13] J. Prost, F. Jülicher, and J.-F. Joanny, Active gel physics, Nat. Phys. 11, 111 (2015).

[14] E. Lauga, Bacterial hydrodynamics, Annu. Rev. Fluid Mech. 48, 105 (2016).

[15] P. Gross, K. V. Kumar, and S. W. Grill, How active mechanics and regulatory biochemistry combine to form patterns in development, Annu. Rev. Biophys. 46, 337 (2017).

[16] A. Cavagna, I. Giardina, and T. S. Grigera, The physics of flocking: Correlation as a compass from experiments to theory, Phys. Rep. 728, 1 (2018).

[17] D. Needleman and Z. Dogic, Active matter at the interface between materials science and cell biology, Nat. Rev. Mater. 2, 17048 (2017).

[18] W. F. Paxton, S. Sundararajan, T. E. Mallouk, and A. Sen, Chemical locomotion, Angew. Chem., Int. Ed. 45, 5420 (2006).

[19] A. M. Menzel, Tuned, driven, and active soft matter, Phys. Rep. 554, 1 (2015).

[20] C. C. Maass, C. Krüger, S. Herminghaus, and C. Bahr, Swimming droplets, Annu. Rev. Condens. Matter Phys. 7, 171 (2016).

[21] W. Wang, X. Lv, J. L. Moran, S. Duan, and C. Zhou, A practical guide to active colloids: Choosing synthetic model systems for soft matter physics research, Soft Matter 16, 3846 (2020).

[22] S. Thutupalli, R. Seemann, and S. Herminghaus, Swarming behavior of simple model squirmers, New J. Phys. 13, 073021 (2011).

[23] Z. Izri, M. N. van der Linden, S. Michelin, and O. Dauchot, Self-Propulsion of Pure Water Droplets by Spontaneous Marangoni-Stress-Driven Motion, Phys. Rev. Lett. 113, 248302 (2014).

[24] J. Palacci, S. Sacanna, A. P. Steinberg, D. J. Pine, and P. M. Chaikin, Living crystals of light-activated colloidal surfers, Science 339, 936 (2013).
[25] N. Kumar, S. Ramaswamy, and A. K. Sood, Symmetry Properties of the Large-Deviation Function of the Velocity of a Self-Propelled Polar Particle, Phys. Rev. Lett. 106, 118001 (2011).

[26] L. Walsh, C. G. Wagner, S. Schlossberg, C. Olson, A. Baskaran, and N. Menon, Noise and diffusion of a vibrated self-propelled granular particle, Soft Matter 13, 8964 (2017).

[27] T. Speck, Collective forces in scalar active matter, Soft Matter 16, 2652 (2020).

[28] W.-F. Hu, T.-S. Lin, S. Rafai, and C. Misbah, Chaotic Swimming of Phoretic Particles, Phys. Rev. Lett. 123, 238004 (2019).

[29] H. C. Berg and D. A. Brown, Chemotaxis in Escherichia coli analysed by three-dimensional tracking, Nature (London) 239, 500 (1972).

[30] M. E. Cates and J. Tailleur, When are active Brownian particles and run-and-tumble particles equivalent? Consequences for motility-induced phase separation, Europhys. Lett. 101, 20010 (2013).

[31] É. Fodor, C. Nardini, M. E. Cates, J. Tailleur, P. Visco, and F. van Wijland, How Far from Equilibrium Is Active Matter? Phys. Rev. Lett. 117, 038103 (2016).

[32] M. Bergert, A. Erzberger, R. A. Desai, I. M. Aspalter, A. C. Oates, G. Charras, G. Salbreux, and E. K. Paluch, Force transmission during adhesion-independent migration, Nat. Cell Biol. 17, 524 (2015).

[33] See Supplemental Material at http://link.aps.org/supplemental/ 10.1103/PhysRevResearch.4.013136 for full details of the experimental setup, data analysis, and theory.

[34] S. Ebbens, R. A. L. Jones, A. J. Ryan, R. Golestanian, and J. R. Howse, Self-assembled autonomous runners and tumblers, Phys. Rev. E 82, 015304(R) (2010).

[35] K. Malakar, V. Jemseena, A. Kundu, K. V. Kumar, S. Sabhapandit, S. N. Majumdar, S. Redner, and A. Dhar, Steady state, relaxation and first-passage properties of a run-andtumble particle in one-dimension, J. Stat. Mech. (2018) 043215.

[36] F. Jülicher, A. Ajdari, and J. Prost, Modeling molecular motors, Rev. Mod. Phys. 69, 1269 (1997).

[37] T. J. Purcell, H. L. Sweeney, and J. A. Spudich, A forcedependent state controls the coordination of processive myosin V, Proc. Natl. Acad. Sci. USA 102, 13873 (2005).

[38] J. C. Liao, J. A. Spudich, D. Parker, and S. L. Delp, Extending the absorbing boundary method to fit dwell-time distributions of molecular motors with complex kinetic pathways, Proc. Nat. Acad. Sci. USA 104, 3171 (2007).

[39] R. Phillips, J. Kondev, J. Theriot, and H. Garcia G, Physical Biology of the Cell, 2nd ed. (Garland Science, London, 2013).

[40] P. Hänggi, P. Talkner, and M. Borkovec, Reaction-rate theory: Fifty years after Kramers, Rev. Mod. Phys. 62, 251 (1990).

[41] B. A. Camley and W.-J. Rappel, Velocity alignment leads to high persistence in confined cells, Phys. Rev. E 89, 062705 (2014).

[42] A. Reversat, F. Gaertner, J. Merrin, J. Stopp, S. Tasciyan, J. Aguilera, I. de Vries, R. Hauschild, M. Hons, M. Piel, A. Callan-Jones, R. Voituriez, and M. Sixt, Cellular locomotion using environmental topography, Nature (London) 582, 582 (2020). 